\title{
Variations on a Theorem of Landau. Part I
}

\section{By Daniel Shanks and Larry P. Schmid}

1. Introduction. Recently [1] we discussed Landau's function $B(x)$ which equals the number of positive integers $\leqq x$ that can be expressed as a sum of two squares $u^{2}+v^{2}$ with $u$ and $v$ nonnegative integers. We showed that

$$
B(x)=\frac{0.764223654 x}{(\log x)^{1 / 2}}\left[1+\frac{0.581948659}{\log x}+O\left(\frac{1}{\log ^{2} x}\right)\right] .
$$

More generally, for any integer $n \neq-k^{2}$, let $B_{n}(x)$ be the number of positive integers $\leqq x$ of the form $u^{2}+n v^{2}$. Then $B_{1}(x)$ is our previous $B(x)$, and it was also shown in [1] that

$$
B_{4}(x)=\frac{0.573167740 x}{(\log x)^{1 / 2}}\left[1+\frac{0.581948659}{\log x}+O\left(\frac{1}{\log ^{2} x}\right)\right] .
$$

For the generalization $B_{n}(x)$ it is known that

$$
B_{n}(x) \sim \frac{b_{n} x}{(\log x)^{1 / 2}}
$$

for some constant $b_{n}$, but to compute this constant is not always easy. As regards the error term in (3) H. H. Ostmann stated, in effect [2], that for $n>0$,

$$
B_{n}(x)=\frac{b_{n} x}{(\log x)^{1 / 2}}+O\left(\frac{x}{\log x}\right),
$$

but this is not always true. For example, it will be seen in the sequel that

$$
B_{14}(x)=\frac{b_{14} x}{(\log x)^{1 / 2}}\left[1-\frac{\beta_{14}}{(\log x)^{1 / 4}}+O\left(\frac{1}{\log x}\right)\right]
$$

for some constant $\beta_{14}$. On the other hand, for many values of $n$ (namely, when there is one class/genus, as will be explained later) Eq. (4) can be strengthened to read

$$
B_{n}(x)=\frac{b_{n} x}{(\log x)^{1 / 2}}\left[1+\frac{c_{n}}{\log x}+O\left(\frac{1}{\log ^{2} x}\right)\right] .
$$

The erroneous (4) stems from a misinterpretation of the results obtained by R. D. James [3] and Gordon Pall [4]. They showed that, for a fixed positive n, the total collection of all positive numbers expressible by at least one form, $a u^{2}+b u v+$ $c v^{2}$ with $b^{2}-4 a c=-4 n$, has a population given by

$$
T_{n}(x)=\frac{t_{n} x}{(\log x)^{1 / 2}}+O\left(\frac{x}{\log x}\right)
$$

for some $t_{n}$. If we have class number 1, all such quadratic forms are equivalent to $u^{2}+n v^{2}$, and (4) follows. But if the class number exceeds unity, (4) does not

Received March 17, 1966. 
automatically follow for the population of an individual form, and in (5), as was stated, the "error" is really of a higher order.

We shall not confine ourselves here either to class number 1 or to definite forms $(n>0)$. In the thesis of Paul Bernays [5], which is referred to in [6] and [7], but which was not available to us, he showed that for any $a, b$, and $c$ such that $b^{2}-4 a c=$ $-4 D \neq k^{2}$, the population of the individual form $a u^{2}+b u v+c v^{2}$ satisfies

$$
P(x) \sim \frac{p_{D} x}{(\log x)^{1 / 2}}
$$

for some $p_{D}$. From this, therefore, one not only has (3), but also the further fact that all forms with the same determinant $D$ have populations that are asymptotic to one another.

If one knew the constant $t_{n}$ of (7), and if the several classes were always disjoint, then one could obtain the $b_{n}$ of (3) merely by dividing $t_{n}$ by the class number. But the classes are not always disjoint and the constants are generally not known. Further, the great variety of second-order terms, as in (5) and (6), has not been investigated. We propose to examine these questions here.

For many values of $n$, with various class numbers, or various group structures with the same class number, and with different types of overlap amongst the several classes, we will evaluate the constants $b_{n}$, indicate the nature of the secondorder term, tabulate the exact populations $B_{n}(x)$, and compare the populations of the different classes.

We thought, at first, to have a subtitle: "Studies in Binary Quadratic Forms" for we must admit that the variety of situations that presented themselves far exceeded our original notions, and required some effort on our part to unravel and understand. Later, we considered whether another alteration of the title was called for, since the investigation, as it developed, was a stimulating one which threw off suggestions in many directions.

Some of these suggestions (only some!) relate to the generalized Riemann Hypothesis, to the exhibition of infinitely many examples having the same class number, to a criterion for Mersenne primes, to a problem of Bateman, to nonalgebraic singularities that can be dominated by algebraic singularities, to trees of prime classes, to the Dirichlet arithmetic progression theorem, and to the failure of factorization using idoneal number techniques. We will present some of these topics as we proceed, interspersing them within the main framework that is called for by the investigation of $B_{n}(x)$.

2. Notation. We have defined $B_{n}(x)$. Similarly, let $B_{a, c}(x)$ equal the population of $a u^{2}+c v^{2}$; we suppress the first subscript if it equals 1 . Further, let $B_{a, b, c}(x)$ be the population of $a u^{2}+b u v+c v^{2}$. And in this last case we must allow $u$ or $v$ to be negative; e.g., $37=4 u^{2}+4 u v+5 v^{2}$ for $(u, v)=(-1,3)$, but 37 is not obtained if the arguments are nonnegative.

Sometimes we are especially interested in the odd (or even) numbers represented by these forms, and we then replace $B$ by $O$ (or $E$ ). Thus $O_{2,3}(x)$ is the number of positive odd integers $\leqq x$ represented by $2 u^{2}+3 v^{2}$. In [1] it was shown that if we have $O_{1}(x)$, this suffices, and we can easily deduce $E_{1}(x)$ and $B_{1}(x)$. One of our tasks here is to determine the extent to which this generalizes for $O_{n}(x)$.

We defined $b_{n}$ in (3) and we defined $c_{n}$ in (6) when (6) is valid. 
We will have much need of the Dirichlet series $L_{n}(s)$ defined by

$$
L_{n}(s)=\sum_{k=0}^{\infty}\left(\frac{-n}{2 k+1}\right)(2 k+1)^{-s}
$$

and its analytic continuation, where $(-n /(2 k+1))$ is the Jacobi symbol, cf. [S]. The function $\zeta_{n}(s)$ is given by

$$
\zeta_{n}(s)=\prod_{r \mid 2 n}\left(1-\frac{1}{r^{8}}\right) \zeta(s)
$$

where the product is taken over all primes $r$ that divide $2 n$, and where $\zeta(s)$ is the Riemann zeta function, cf. [9]. This function $\zeta_{n}(s)$ can always be written as an $L$ function since it equals $L_{-m^{2}}(s)$ where $m$ is the product of all distinct odd primes dividing $n$. Whichever notation is more convenient will be used in a particular instance.

The quantities $g_{n}$ are also needed. Here

$$
g_{n}=\prod_{q}\left(1-q^{-2}\right)^{-1 / 2}
$$

where the $q$ 's are all the odd primes that have $-n$ as a quadratic nonresidue:

$$
(-n / q)=-1
$$

If the primes $p$ satisfy

$$
(-n / p)=+1
$$

then

$$
\zeta_{n}(s)=\prod_{p, q} 1 /\left(1-1 / p^{s}\right)\left(1-1 / q^{s}\right)
$$

while

$$
L_{n}(s)=\prod_{p, q} 1 /\left(1-1 / p^{s}\right)\left(1+1 / q^{s}\right)
$$

Therefore

$$
\frac{\zeta_{n}(s)}{L_{n}(s)}=\prod_{q} \frac{1+1 / q^{s}}{1-1 / q^{s}}=\prod_{q} \frac{1-1 / q^{2 s}}{\left(1-1 / q^{8}\right)^{2}}
$$

or

$$
\prod_{q} \frac{1}{1-1 / q^{s}}=\left(\frac{\zeta_{n}(s)}{L_{n}(s)}\right)^{1 / 2} \prod_{q} \frac{1}{\left(1-1 / q^{2 s}\right)^{1 / 2}}
$$

It follows, by induction, that $g_{n}$ may be evaluated by the very rapidly convergent product

$$
g_{n}=\left(\frac{\zeta_{n}(2)}{L_{n}(2)}\right)^{1 / 4}\left(\frac{\zeta_{n}(4)}{L_{n}(4)}\right)^{1 / 8}\left(\frac{\zeta_{n}(8)}{L_{n}(8)}\right)^{1 / 16} \cdots
$$

provided that the constants $L_{n}\left(2^{k}\right)$ are available. The formula in (15) may be nested:

$$
g_{n}=\sqrt[4]{ }\left\{\left[\zeta_{n}(2) / L_{n}(2)\right] \sqrt{ }\left\{\left[\zeta_{n}(4) / L_{n}(4)\right] \sqrt{ }\{\cdots\}\right\}\right\},
$$

and for the accuracies aimed at here three terms generally suffice. 
We also need the generating functions $f_{n}(s)$ (or $f_{a, c}(s)$, or $\left.f_{a, b, c}(s)\right)$ where

$$
f_{n}(s)=\sum_{k=1}^{\infty} a_{k} k^{-s}
$$

with $a_{k}=1$ or 0 according as $k$ is, or is not, representable by $u^{2}+n v^{2}$, (or $a u^{2}+c v^{2}$, etc.). For example, cf. [1, p. 81$]$,

$$
f_{1}(s)=\frac{1}{1-1 / 2^{s}} \prod_{p} \frac{1}{1-1 / p^{s}} \prod_{q} \frac{1}{1-1 / q^{2 s}}
$$

where the primes $p \equiv 1(\bmod 4)$ and the primes $q \equiv-1(\bmod 4)$.

If $n$ corresponds to a large class number, or to many genera, or if it has square factors, $N^{2} \mid n$, or if we have some combination of these, $f_{n}(s)$ may be much more complicated. This creates one of our main tasks.

It will be convenient to introduce an abbreviated notation. Let $[P]$ be given by

$$
[P]=\prod_{p} \frac{1}{1-1 / p^{s}}
$$

the product being taken over some class of primes $P$. Further let [2], say, or $[-P]$, or $\left[Q^{2}\right]$ be given by

$$
\frac{1}{1-1 / 2^{s}}, \quad \prod_{p} \frac{1}{1+1 / p^{s}}, \quad \prod_{q} \frac{1}{1-1 / q^{2 s}}
$$

respectively. A subscript, such as in $[Q]_{2}$, will mean that $s=2$. Thus (11) and (17) may be written

$$
g_{n}=\left([Q]_{2}\right)^{1 / 2}
$$

and

$$
f_{1}(s)=[2][P]\left[Q^{2}\right] .
$$

In what follows, whenever we are discussing $B_{n}(x)$, by $P$ we mean the class of primes $p$ that have $-n$ as a quadratic residue, while $Q$ is the class of primes $q$ that have $-n$ as a quadratic nonresidue:

$$
(-n / p)=+1, \quad(-n / q)=-1 .
$$

3. Class Number 1. We start with the easier cases. For $n=1$, a number $m$ can be expressed as $m=u^{2}+v^{2}$ if and only if in its factorization into primes, namely

$$
m=2^{\alpha} \prod p_{i}^{\alpha_{i}} \prod q_{i}{ }^{2 \beta_{i}}
$$

we find an arbitrary power of $2, \alpha \geqq 0$, arbitrary powers of any number of primes $p_{i} \equiv 1(\bmod 4)$ and arbitrary even powers of any number of primes $q_{i} \equiv-1(\bmod 4)$. Our abbreviated expression for the generator $f_{1}(s)$, in $(20)$, indicates this symbolically.

Suppose for some $n$ every $p$ in $P$ can be expressed as $u^{2}+n v^{2}$; that is, assume that

$$
(-n / p)=1 \text { implies } p=u^{2}+n v^{2} .
$$


Then we also have class number 1 , as in the case $n=1$. This occurs for $n=7,4,3$, $2,1,-2,-5,-13,-17,-29,-41,-53,-61,-73,-\$ 9,-97$ and some larger negative primes. In our choice of examples, however, we will largely confine ourselves, here and later, to the 200 values of $n \neq-k^{2}$ that satisfy $|n| \leqq 105$. Naturally, we will not examine in detail even all of these; in fact, some of them are quite complicated.

The 16 class number 1 cases mentioned above have four different types of behavior.

(a) $n=-2,1,2$.

Here $u^{2}+n v^{2}$ not only represents every prime $p$ in $P$ but also represents the prime 2 .

(b) $n=-61,-53,-29,-13,-5,3$.

Here $n$ can be certain positive or negative primes $\equiv 3(\bmod 8)$. For some other such primes, e.g., $n=-101,-37,+11,+19$, etc. the class number is 3 or larger. Some of those cases are examined later. In this case (b) $2^{\alpha}$ is represented by $u^{2}+n v^{2}$ if and only if $\alpha$ is even. In other words, the prime 2 acts like a prime $q$ here in distinction to its behavior in case (a) where it acts like a prime $p$. But the remaining prime $|n|$ is again representable, and thus behaves like a $p$.

(c) $n=-97,-89,-73,-41,-17,7$.

Here $n$ can be certain positive or negative primes $\equiv 7(\bmod 8)$, but again not every such prime since $-257,+23$, +31 , etc. have class number 3 or larger. This time $2^{\alpha}=u^{2}+n v^{2}$ if and only if $\alpha \geqq 2$. While 2 is not representable, 8 is. Specifically :

$S=1^{2}+7 \cdot 1^{2}=5^{2}-17 \cdot 1^{2}=7^{2}-41 \cdot 1^{2}=9^{2}-73 \cdot 1^{2}$

$$
=217^{2}-89 \cdot 23^{2}=69^{2}-97 \cdot 7^{2} .
$$

The above assertion follows since 4 is obviously representable as $4=2^{2}+n \cdot 0^{2}$, and all higher powers of 2 can be written as $4^{k}$ or $8 \cdot 4^{k}$. Again, $|n|$ is representable.

(d) $n=4$.

Here, as in (c), $2^{\alpha}$ is representable if and only if $\alpha \geqq 2$, but the main difference here is that $n$ is now divisible by a square $>1$. These cases will be treated separately as we proceed.

It follows that the generating functions in these four cases are given by the following four formulas.

$$
\begin{aligned}
f_{n}(s) & =[2][P]\left[Q^{2}\right], \\
f_{n}(s) & =[4][|n|][P]\left[Q^{2}\right], \\
f_{n}(s) & =\left(1-1 / 2^{s}+1 / 4^{s}\right)[2][|n|][P]\left[Q^{2}\right], \\
f_{4}(s) & =\left(1-1 / 2^{s}+1 / 4^{s}\right)[2][P]\left[Q^{2}\right] .
\end{aligned}
$$

Now, for any $n$, we have

$$
\zeta_{n}(s) L_{n}(s)=[P]^{2}\left[Q^{2}\right]
$$

from (12) and (13). Therefore, the common factor $[P]\left[Q^{2}\right]$ in (22) may be written

$$
[P]\left[Q^{2}\right]=\left(\zeta_{n}(s) L_{n}(s)\right)^{1 / 2}\left(\left[Q^{2}\right]\right)^{1 / 2},
$$


and using (10) we have

$$
[P]\left[Q^{2}\right]=\prod_{r \mid 2 n}\left(1-1 / r^{s}\right)^{1 / 2}\left(\zeta(s) L_{n}(s)\right)^{1 / 2}\left(\left[Q^{2}\right]\right)^{1 / 2} .
$$

Since $\zeta(s)$ has the principal part: $1 /(s-1)$, at $s=1$, and the remaining factors in (24) and (22) are analytic at $s=1$, any one of the generating functions in (22) may be expanded as follows.

$$
f_{n}(s)=D_{n} /(s-1)^{1 / 2}\left[1+\alpha_{1, n}(s-1)+\alpha_{2, n}(s-1)^{2}+\cdots\right]
$$

where the constant $D_{n}$ has the common factor

$$
\prod_{r \mid 2 n}(1-1 / r)^{1 / 2}\left(L_{n}(1)\right)^{1 / 2} g_{n}
$$

from (24) and (11), and this is to be multiplied by

$$
2, \quad \frac{4}{3}|n| /(|n|-1), \quad \frac{3}{2}|n| /(|n|-1), \quad \text { or } \quad \frac{3}{2},
$$

depending on whether we have case (a), (b), (c), or (d), respectively.

Alternatively, we may use Euler's $\phi$ function and may verify that

$$
D_{n}=\delta_{n}\left[L_{n}(1) \cdot 2|n| / \phi(2|n|)\right]^{1 / 2} g_{n}
$$

where $\delta_{n}$ is given by

respectively.

$$
\delta_{n}=1, \frac{2}{3}, \frac{3}{4}, \text { or } \frac{3}{4}
$$

From the generator (25) the analysis of $B_{n}(x)$ in these cases proceeds almost exactly as in Landau's original paper on $B_{1}(x)$ (see [10], or cf. [1]), the main difference being that the $L(s)=L_{1}(s)$ there is now replaced by $L_{n}(s)$. One, therefore, also obtains equation (6) in these cases, but with the coefficient now given by

$$
b_{n}=D_{n} / \Gamma\left(\frac{1}{2}\right)=\delta_{n} g_{n}\left[L_{n}(1) \cdot 2|n| / \pi \phi(2|n|)\right]^{1 / 2} .
$$

The constants $c_{n}$ in (6) are more difficult to compute; we will not calculate them here, but for $n>0$ a computation analogous to that for $c_{1}$ in [1] is possible since $L_{n}{ }^{\prime}(1) / L_{n}(1)$ can be evaluated in terms of gamma functions when $n$ is positive.

4. Calculation of $b_{n}$. First Digression. Since $L_{n}(1)$ is expressible in closed form, cf. [8], the only problem in evaluating $b_{n}$ from (27) is that of computing the quantity $g_{n}$. The product in (11) converges very poorly, and if one must depend upon this an accurate value of $b_{n}$ is not obtainable. We will see later that this is the case for some interesting examples: $n=11,14,21$, etc. But if the numbers $L_{n}\left(2^{k}\right)$ are available, say, for $k=1,2,3$, the very rapidly convergent (15) may be used.

Now, for any $n<0, L_{n}(2 m)$ is available in closed form (at least in principle) for all even integers $2 m,[8]_{n}$ For any negative $n$, therefore, (15) enables us to compute $g_{n}$ accurately. For some positive values of $n$, including $1 \leqq n \leqq 10$, the $L_{n}(2 m)$ have been computed numerically, [8], [11], and (15) again may be used. It follows that in all our 16 class number 1 cases, we could compute $b_{n}$ accurately from (27) and (15), although for the larger values of $n$, such as -97 and -89 , the computation would be tedious. We include within a larger Table 1 accurate values of $b_{n}$ for $n=-17,-5,-2,1,2,3,4$, and 7 selected from the 16 cases presently under consideration. 
TABLE 1

Constants

\begin{tabular}{l|l|l}
$b_{-34}=0.505360417$ & $b_{2}=0.872887558$ & $b_{12}=0.399318378$ \\
$b_{-17}=0.484644756$ & $b_{3}=0.638909405$ & $b_{13} \approx 0.420(6)$ \\
$b_{-10}=0.488162034$ & $b_{4}=0.573167740$ & $b_{14} \approx 0.563(5)$ \\
$b_{-8}=0.344664285$ & $b_{5}=0.535179999$ & $b_{16}=0.334347848$ \\
$b_{-7}=0.455065213$ & $b_{6}=0.558357114$ & $b_{20}=0.401384999$ \\
$b_{-6}=0.482889041$ & $b_{7}=0.543539641$ & $b_{24}=0.279178557$ \\
$b_{-5}=0.515939482$ & $b_{8}=0.436443779$ & $b_{27}=0.496929538$ \\
$b_{-3}=0.441875842$ & $b_{9}=0.424568696$ & $b_{64}=0.274642876$ \\
$b_{-2}=0.689328571$ & $b_{10}=0.473558100$ & $b_{96}=0.209383918$ \\
$b_{1}=0.764223654$ & $b_{11} \approx 0.677(4)$ & $b_{256}=0.259716632$
\end{tabular}

We illustrate the foregoing discussion by a computation of $b_{-17}$, a number that we will need later anyway. We have, from (27),

$$
b_{-17}=\frac{3}{4} g_{-17}\left(34 L_{-17}(1) / 16 \pi\right)^{1 / 2} .
$$

Here we want the closed formula [8]:

$$
\begin{aligned}
L_{-17}(1) & =17^{-1 / 2} \log \left(4+17^{1 / 2}\right)=17^{-1 / 2} \log \left[8+\frac{1}{8}+\frac{1}{8}+\cdots\right] \\
& =0.5080424169 .
\end{aligned}
$$

To compute $g_{n}$ for negative $n$ in the manner indicated above we note that, for negative $n, \zeta_{n}(2 k)$ and $L_{n}(2 k)$ can be expressed [8] as:

$$
\zeta_{n}(2 k)=r_{n, k} \pi^{2 k}, \quad L_{n}(2 k)=s_{n, k} \pi^{2 k} /(-n)^{1 / 2}
$$

for computable rational numbers $r_{n, k}$ and $s_{n, k}$. Thus, in our case, we obtain

$$
\frac{\zeta_{-17}(2)}{L_{-17}(2)}=\frac{6}{\sqrt{ } 17}, \quad \frac{\zeta_{-17}(4)}{L_{-17}(4)}=\frac{174}{41 \sqrt{ } 17}, \quad \frac{\zeta_{-17}(8)}{L_{-17}(8)}=\frac{123529038}{29950897 \sqrt{ } 17},
$$

and therefore, from the rapidly convergent product (15), we find

$$
g_{-17}=1.102320127 \text {. }
$$

Finally we compute

$$
b_{-17}=0.484644756 .
$$

We digress here to note that the Eqs. (28) can be used to give a simple, attractive proof of a known

Theorem. If $a>0$ and $\neq k^{2}$ then a is a quadratic residue for infinitely many primes and a quadratic nonresidue for infinitely many.

Proof. For such an $a$, from (12), (13), and (28), we have

and

$$
\frac{\zeta_{-a}(2)}{L_{-a}(2)}=\prod_{q} \frac{q^{2}+1}{q^{2}-1}=k_{a} a^{1 / 2}
$$

$$
\frac{\zeta_{-a}(2) L_{-a}(2)}{\zeta_{-a}(4)}=\prod_{p} \frac{p^{2}+1}{p^{2}-1}=l_{a} a^{1 / 2}
$$


for rational numbers $k_{a}$ and $l_{a}$, with the products taken over all primes $q$ and $p$ such that $(a \mid q)=-1$ and $(a \mid p)=+1$. If the theorem is assumed false we obtain a contradiction, since for these values of $a, a^{1 / 2}$ is irrational.

Returning to $b_{n}$, we also note that it is easier to compute a rough value of $b_{n}$ for $n>0$ than for $n<0$, but much more difficult to compute an accurate value when $n>0$. This is because the $L_{n}(1)$ are simpler if $n>0$, e.g., $L_{7}(1)=\pi / 2 \sqrt{ } 7$, and, for all $n, g_{n}$ is larger than, but close to, unity. Thus $b_{7}$ is bounded below by the simply computed $(252)^{1 / 4} / 8=0.498$. On the other hand, for $n>0$ the $L_{n}\left(2^{k}\right)$ are not available in closed form, and therefore $g_{n}$ is much harder to compute accurately.

We might note that while James [3] and Pall [4] confined themselves to definite forms, in this matter of the accurate computation of the $b_{n}$, the indefinite forms are easier. Further, as already stressed by Gauss [15], when $n<0$ we encounter many more examples of small class number, and of small number of classes/genus, both of which conditions greatly simplify the theory. We already noted above more class number 1 cases with $n<0$, and for other relatively simple cases this predominance becomes even greater.

5. Calculation of $B_{n}$. Some Complications. The exact values of $B_{n}(x)$ given in the tables here were obtained by the second-named author with an IBM 7090 . For the values of $n$ examined the average program ran 10 minutes.

In the efficient computation of $B_{1}(x)$ discussed in [1, p. 81] we merely counted the population of the odd numbers, $O_{1}(x)$, and deduced the values of $E_{1}(x)$ and $B_{1}(x)$ by the simple recurrence relations:

$$
\begin{aligned}
& E_{1}(x)=E_{1}(x / 2)+O_{1}(x / 2), \\
& B_{1}(x)=E_{1}(x)+O_{1}(x) .
\end{aligned}
$$

Since these odd numbers are given by $m^{2}+4 M^{2}$, where $m$ goes through the odd integers and $M$ goes through all integers, one successively adds $1+8+16+$ $24+\cdots$ to a fixed $4 M^{2}$ and records, by a mark, all such sums. It develops, therefore, that the program consists almost exclusively of counting and "logical" operations.

The generalization of that program used here for $B_{n}(x)$ computed $A m^{2}+B M^{2}$ for fixed integers $A$ and $B$ and for $m$ odd, as before. For $B_{2}(x)$ everything proceeds as before by computing $m^{2}+2 M^{2}$. But for $B_{3}(x)$ two modifications are necessary.

Firstly, there are two types of odd numbers of the form $u^{2}+3 v^{2}$, those with $v$ odd, and those with $v$ even:

$$
3 m^{2}+4 M^{2} \text { and } m^{2}+12 M^{2} .
$$

As may be seen, these two sets are disjoint so that we have

$$
O_{3}(x)=O_{3,4}(x)+O_{12}(x) .
$$

One therefore computes the two counts on the right separately, and then merely adds. A similar problem arises for any $B_{n}(x)$ with odd $n$, but while a similar solution is available for, say, $n=7$, with

$$
O_{7}(x)=O_{7,4}(x)+O_{28}(x)
$$


for $n=5$ this device fails, since

$$
5 m^{2}+4 M^{2} \text { and } m^{2}+20 M^{2}
$$

are not disjoint, and have instead a complicated intersection. This mysterious intersection becomes clarified only after we have analyzed the cyclic class number 4 cases. Similarly, while one immediately observes that the counts $O_{3,4}(x)$ and $O_{12}(x)$ are nearly equal, this only becomes understandable after we study class number 2 . The desire to attain greater computing efficiency here, by counting only the odd numbers, therefore forces us to examine these interesting phenomena.

A second modification needed in computing some $B_{n}(x)$ in this way stems from the fact (page 555) that factors of $2^{\alpha}$ may occur according to different laws, and consequently that the recurrence in (29) may need to be altered. For $n=3$, or generally for type (b) of page 555 , one must replace the first line of (29) by

$$
E_{n}(x)=E_{n}(x / 4)+O_{n}(x / 4),
$$

while for $n=4$, or generally for types (c) or (d), one uses

$$
E_{n}(x)=E_{n}(x / 2)+O_{n}(x / 4) \text {. }
$$

Still another type of recurrence is needed for certain class number 2 cases such as $n=6$, but we postpone that until later.

A third complication in computing $B_{n}(x)$ occurs for all negative $n$. Writing $n=-N$ temporarily we consider the indefinite form:

$$
u^{2}-N v^{2}=m \text {. }
$$

Our previous procedure, of determining (in effect) all solutions of $u^{2}+n v^{2}=m$ for all (positive, odd) values of $m \leqq x$ is no longer feasible. For now if $m$ has any solution $(u, v)$ in $(30)$, it has infinitely many solutions. The algorithm may be kept finite, however, by the use of Theorem 108 in Nagell [12]:

If $u+v N^{1 / 2}$ is a fundamental solution of (30) and $x+y N^{1 / 2}$ is the fundamental solution of

$$
x^{2}-N y^{2}=1
$$

then

$$
\begin{aligned}
& 0 \leqq v \leqq m^{1 / 2} y /(2(x+1))^{1 / 2}, \\
& 0<|u| \leqq[(x+1) m / 2]^{1 / 2} .
\end{aligned}
$$

For any $m$ it follows that we can restrict the variable $v$ to those satisfying

$$
v^{2} \leqq m y^{2} / 2(x+1),
$$

and, for any fixed $v$, we can insist upon

$$
u \geqq v(x+1) / y \text {. }
$$

For example, since the fundamental solutions of (31) for $N=2$ and $N=34$ are $3+2 \sqrt{ } 2$ and $35+6 \sqrt{ } 34$ respectively, we can confine ourselves to $v^{2} \leqq m / 2$, together with

in these two cases.

$$
u \geqq 2 v \quad \text { and } \quad u \geqq 6 v
$$


TABLE 2

Populations, Class No. 1

\begin{tabular}{|c|c|c|c|c|c|}
\hline$x$ & $B_{1}(x)$ & $B_{2}(x)$ & $B_{3}(x)$ & $B_{4}(x)$ & $B_{-2}(x)$ \\
\hline $2^{0}$ & 1 & 1 & 1 & 1 & 1 \\
\hline $2^{1}$ & 2 & 2 & 1 & 1 & 2 \\
\hline $2^{2}$ & 3 & 4 & 3 & 2 & 3 \\
\hline $2^{3}$ & 5 & 6 & 4 & 4 & 5 \\
\hline $2^{4}$ & 9 & 10 & S & 7 & 8 \\
\hline $2^{5}$ & 16 & 18 & 14 & 12 & 15 \\
\hline $2^{6}$ & 29 & 33 & 25 & 22 & 26 \\
\hline $2^{7}$ & 54 & 60 & 45 & 41 & 48 \\
\hline $2^{8}$ & 97 & 111 & 82 & 72 & 87 \\
\hline $2^{9}$ & 180 & 205 & 151 & 137 & 161 \\
\hline $2^{10}$ & 337 & 385 & 282 & 254 & 299 \\
\hline $2^{11}$ & 633 & 725 & 531 & 476 & 563 \\
\hline $2^{12}$ & 1197 & 1374 & 1003 & 901 & 1066 \\
\hline $2^{13}$ & 2280 & 2610 & 1907 & 1716 & 2030 \\
\hline $2^{14}$ & 4357 & 4993 & 3645 & 3274 & 3885 \\
\hline $2^{15}$ & 8363 & 9578 & 6993 & 6286 & 7464 \\
\hline $2^{16}$ & 16096 & 18426 & 13456 & 12090 & 14384 \\
\hline $2^{17}$ & 31064 & 35568 & 25978 & 23331 & 27779 \\
\hline $2^{18}$ & 60108 & 68806 & 50248 & 45140 & 53782 \\
\hline $2^{19}$ & 116555 & 133411 & 97446 & 87511 & 104359 \\
\hline $2^{20}$ & 226419 & 259145 & 189291 & 169972 & 202838 \\
\hline $2^{21}$ & 440616 & 504222 & 368338 & 330752 & 394860 \\
\hline $2^{22}$ & 858696 & 982538 & 717804 & 644499 & 769777 \\
\hline $2^{23}$ & 1675603 & 1917190 & 1400699 & 1257523 & 1502603 \\
\hline $2^{24}$ & 3273643 & 3745385 & 2736534 & 2456736 & 2936519 \\
\hline 225 & 6402706 & 7324822 & 5352182 & 4804666 & 5744932 \\
\hline 2 & 12534812 & & 10478044 & 9405749 & \\
\hline
\end{tabular}

We note, then, that the fundamental solution of (31) is needed not only in ( valuating the number $L_{-n}(1)$ (which is used in computing the constant $b_{-n}$ ) but it is also needed here in computing $B_{-n}(x)$.

6. First Table of $B_{n}(x)$. Comparisons. In Table 2 we list values of $B_{n}(x)$ for tive class number 1 cases, and for arguments $x=2^{k}$ up to $x=2^{25}=33554432$ or $x=2^{26}=67108864$. An interested reader can easily recover the corresponding values of $O_{n}(x)$ and $E_{n}(x)$, if he wishes, by use of the recurrences $(29,29 \mathrm{a}, 29 \mathrm{~b})$.

From (6) we have

$$
\frac{B_{n}(x)}{B_{1}(x)}=\frac{b_{n}}{b_{1}}\left[1+\frac{c_{n}-c_{1}}{\log x}+O\left(\frac{1}{\log ^{2} x}\right)\right] .
$$

One finds from Tables 1 and 2 that $B_{2}(x) / B_{1}(x)$ approaches $b_{2} / b_{1}$ from above, while $B_{-2}(x) / B_{1}(x)$ approaches $b_{-2} / b_{1}$ from below. This implies that

$$
c_{2}>c_{1}=0.581948659>c_{-2}
$$

but we have not computed $c_{ \pm 2}$ to verify this. $B_{3}(x)$ remains so closely proportional to $B_{1}(x)$ that it is not clear from this data whether $c_{3}>c_{1}$ or $c_{3}<c_{1}$. It would be 
unlikely that they are exactly equal. But $c_{4}=c_{1}$, as is known [1, Theorem 2]. In fact, we have the theoretical relation

$$
\frac{B_{4}(x)}{B_{1}(x)}=\frac{3}{4}\left[1+\frac{1 / 4}{\left(\log _{2} x\right)^{2}}+\frac{1.46457444}{\left(\log _{2} x\right)^{3}}+O\left(\frac{1}{\left(\log _{2} x\right)^{4}}\right)\right] .
$$

The column $B_{4}(x)$ in Table 2 is actually redundant there since one has

$$
B_{4}(x)=B_{1}(x)-B_{1}(x / 2)+B_{1}(x / 4) \text {. }
$$

We note, in passing, that of all binary forms $u^{2}+n v^{2}, u^{2}+2 v^{2}$ is the most populous, since $b_{2}$ is the largest of these constants. Similarly, it is of interest that $u^{2}+v^{2}+2 w^{2}$ appears to be the most populous of all ternary forms $u^{2}+v^{2}+n w^{2}$. However, there is no exact correlation here since $u^{2}+v^{2}+n w^{2}$ represents 20/24, $22 / 24,21 / 24,17 / 24,20 / 24$, or $21 / 24$ of all numbers when $n$ equals 1 to 6 , respectively, and this is not quite in the same order as the magnitudes of $b_{n}$. The theory appears to be incomplete; e.g., for $n=10$ the fraction is unknown [13].

7. Class Number 2 and the Generalized Riemann Hypothesis. There are 64 class number 2 cases in the range $-105 \leqq n \leqq 105$. All of these are characterized by the fact that the primes $p$ in $P$ fall into two (equinumerous) classes, those in a class $P_{0}$ that are representable by the form $F_{0}: u^{2}+n v^{2}$, and those in a second class $P_{1} n o t$ representable by $F_{0}$, but which are representable by another form $F_{1}: a u^{2}$ $+b u v+c v^{2}$ with $b^{2}-4 a c=-4 n$. Consider first the 32 cases where $\pm n$ is a prime, $\pi$, or twice a prime, $2 \pi$. The behavior here is analogous to the class number 1 type (a) in Section 3, but already there are more variations. We may have different formulas for $F_{1}$ and differing ways of representing the prime divisors of $2 n$. If such a prime divisor is representable by $F_{0}$ we say it is in $P_{0}{ }^{\prime}$, while if it is representable by $F_{1}$ we say it is in $P_{1}^{\prime}$. One, and only one, of these representations is possible. In Tables 3 and 4 we show the variations that occur. Thus, for $n=5,5$ is representable by $u^{2}+5 v^{2}$ (obviously), and 2 is representable by $2 u^{2}+2 u v+3 v^{2}$ (also obviously).

For all class number 2 cases let the two classes of all positive numbers representable by $F_{0}$ and $F_{1}$ be $\Phi_{0}$ and $\Phi_{1}$. Under composition these two classes satisfy the group multiplication of the group of order two:

with

$$
\Phi_{i} \Phi_{j}=\Phi_{k}
$$

$$
k \equiv i+j(\bmod 2) \text {. }
$$

TABLE 3

Variations, Class No. 2

\begin{tabular}{c|c}
\hline Type & \multicolumn{1}{|c}{ Values of $n$} \\
\hline$a_{1}$ & $5,13,37$ \\
$a_{2}$ & $-86,-38,-22,-6$ \\
$a_{3}$ & $-83,-67,-59,-43,-19,-11,-3$ \\
$a_{4}$ & $-74,-58,-26,-10$ \\
$a_{5}$ & $-103,-71,-47,-31,-23,-7$ \\
$a_{6}$ & $-94,-62,-46,-14$ \\
$a_{7}$ &
\end{tabular}


TABLE 4

Behavior in the Different Types

\begin{tabular}{c|c|c|c|c|c}
\hline Type & $\begin{array}{c}\text { Form } \\
\text { of } n\end{array}$ & \multicolumn{1}{|c|}{$F_{0}$} & $F_{1}$ & $P_{0}^{\prime}$ & $P_{1}^{\prime}$ \\
\cline { 2 - 4 } & $\pi$ & $u^{2}+\pi v^{2}$ & $2 u^{2}+2 u v+\left(\frac{\pi+1}{2}\right) v^{2}$ & $\pi$ & 2 \\
$\mathrm{a}_{1}$ & $\pi$ & $2 \pi u^{2}-v^{2}$ & $\pi$ & 2 \\
$\mathrm{a}_{2}$ & $-2 \pi$ & $u^{2}-2 \pi v^{2}$ & $\pi u^{2}-v^{2}$ & - & $2, \pi$ \\
$\mathrm{a}_{3}$ & $-\pi$ & $u^{2}-\pi v^{2}$ & $2 u^{2}+\pi v^{2}$ & - & $2, \pi$ \\
$\mathrm{a}_{4}$ & $2 \pi$ & $u^{2}+2 \pi v^{2}$ & $2 u^{2}-\pi v^{2}$ & - & $2, \pi$ \\
$\mathrm{a}_{5}$ & $-2 \pi$ & $u^{2}-2 \pi v^{2}$ & $\pi u^{2}-v^{2}$ & 2 & $\pi$ \\
$\mathrm{a}_{6}$ & $-\pi$ & $u^{2}-\pi v^{2}$ & $2 \pi u^{2}-v^{2}$ & 2 & $\pi$ \\
$\mathrm{a}_{7}$ & $-2 \pi$ & $u^{2}-2 \pi v^{2}$ & & &
\end{tabular}

It follows that a positive number prime to $2 n$ is in $\Phi_{0}$ or $\Phi_{1}$, according as the total number of its prime factors which are contained in $P_{1}$ (counting multiplicity) is even or odd. Therefore the subsets of the numbers in $\Phi_{0}$ and $\Phi_{1}$ prime to $2 n$ have generating functions:

$$
\begin{aligned}
& \frac{1}{2}\left[P_{0}\right]\left[Q^{2}\right]\left\{\left[P_{1}\right]+\left[-P_{1}\right]\right\}, \\
& \frac{1}{2}\left[P_{0}\right]\left[Q^{2}\right]\left\{\left[P_{1}\right]-\left[-P_{1}\right]\right\}
\end{aligned}
$$

for $\Phi_{0}$ and $\Phi_{1}$ respectively. The generating functions for all members of these sets are obtained from (35) simply by including factors for those primes in $P_{0}{ }^{\prime}$ or $P_{1}^{\prime}$ alongside the corresponding factors $\left[P_{0}\right]$ or $\left[P_{1}\right]$. Thus, for $n=5$, type $a_{1}$, we have the generators

$$
\frac{1}{2}\left[P_{0}\right][5]\left[Q^{2}\right]\left\{[2]\left[P_{1}\right] \pm[-2]\left[-P_{1}\right]\right\}
$$

where we take the $+\operatorname{sign}$ for $f_{5}(s)$ and the $-\operatorname{sign}$ for $f_{2,2,3}(s)$. Similarly, for $n=6$, type $a_{4}$, we have

$$
\frac{1}{2}\left[P_{0}\right]\left[Q^{2}\right]\left\{[2][3]\left[P_{1}\right] \pm[-2][-3]\left[-P_{1}\right]\right\}
$$

for $f_{6}(s)$ and $f_{2,3}(s)$ respectively.

In all these generators the function $[P]=\left[P_{0}\right]\left[P_{1}\right]$ has the same type of singularity at $s=1$ as we discussed for class number 1 and, in fact, (24) remains valid. But the function

$$
\left[P_{0}\right]\left[-P_{1}\right]=\prod_{p \in P_{0}} \frac{1}{1-1 / p^{s}} \prod_{p \leqslant P_{1}} \frac{1}{1+1 / p^{s}},
$$

on the contrary, is analytic at $s=1$ owing to the fact, proven by Landau [14], that the classes $P_{0}$ and $P_{1}$ are equinumerous. For example, for $n=6$, the primes in $P_{0}$ are those where the characters $(2 / p)$ and $(-3 / p)$ are both +1 , while those in $P_{1}$ have both characters equal to -1 . From this we find that

$$
L_{-2}(s) L_{3}(s)=\left[P_{0}\right]^{2}\left[-P_{1}\right]^{2}\left[Q^{2}\right] .
$$

Therefore the $\left[P_{0}\right]\left[-P_{1}\right]\left[Q^{2}\right]$ in the second term of $(36)$ is given by

$$
\left(L_{-2}(s) L_{3}(s)\left[Q^{2}\right]\right)^{1 \cdot 2} \text {, }
$$


TABLE 5

Populations, Class No. 2

\begin{tabular}{|c|c|c|c|c|}
\hline$x$ & $B_{6}(x)$ & $B_{2,3}(x)$ & $B_{10}(x)$ & $B_{2,5}(x)$ \\
\hline $2^{0}$ & 1 & 0 & 1 & 0 \\
\hline $2^{1}$ & 1 & 1 & 1 & 1 \\
\hline $2^{2}$ & 2 & 2 & 2 & 1 \\
\hline $2^{3}$ & 4 & 4 & 2 & 4 \\
\hline $2^{4}$ & 8 & 7 & $\overline{7}$ & $\tilde{j}$ \\
\hline $2^{5}$ & 13 & 14 & 10 & 11 \\
\hline $2^{6}$ & 24 & 23 & 20 & 20 \\
\hline $2^{7}$ & 42 & 42 & 36 & 36 \\
\hline $2^{8}$ & 76 & 76 & 65 & 65 \\
\hline $2^{9}$ & 140 & 139 & 118 & 119 \\
\hline $2^{10}$ & 257 & 258 & 221 & 218 \\
\hline $2^{11}$ & 483 & 482 & 409 & 412 \\
\hline $2^{12}$ & 907 & 907 & 776 & 770 \\
\hline $2^{13}$ & 1717 & 1717 & 1463 & 1466 \\
\hline $2^{14}$ & 3272 & 3269 & 2788 & 2784 \\
\hline $2^{15}$ & 6261 & 6257 & 5328 & 5322 \\
\hline $2^{16}$ & 12027 & 12020 & 10222 & 10226 \\
\hline $2^{17}$ & 23172 & 23171 & 19714 & 19691 \\
\hline $2^{18}$ & 44769 & 44762 & 38054 & 38048 \\
\hline $2^{19}$ & 86708 & 86683 & 73685 & 73665 \\
\hline $2^{20}$ & 168245 & 168233 & 142944 & 142927 \\
\hline $2^{21}$ & 327073 & 327053 & 277838 & 277822 \\
\hline $2^{22}$ & 636849 & 636837 & 540889 & 540851 \\
\hline $2^{23}$ & 1241720 & 1241723 & 1054535 & 1054502 \\
\hline $2^{24}$ & 2424290 & 2424228 & 2058537 & 2058507 \\
\hline $2^{25}$ & 4738450 & 4738426 & 4023278 & 4023164 \\
\hline
\end{tabular}

which is analytic at $s=1$, and remains analytic for the real part of $s>\frac{1}{2}$ provided that the extended Riemann Hypothesis is true.

In any case, from the known zero-free regions of the $L$ functions, the contribution of this second term in (36) cannot modify the counts $B_{6}(x)$ and $B_{2,3}(x)$ by more than $o\left(x \log ^{-m} x\right)$ for any $m$. If $L_{-2}(s)$ and $L_{3}(s)$ satisfy the Riemann Hypothesis then this contribution is $o\left(x^{1 / 2+\epsilon}\right)$ for any positive $\epsilon$.

It follows in this case, and similarly in all 32 cases in Table 3, that (6) is again valid and that the formula for $b_{n}$ in $(27)$, with $\delta_{n}=1$, must be altered only by the factor $\frac{1}{2}$ in $(35)$ :

$$
b_{n}=\frac{1}{2} g_{n}\left[L_{n}(1) \cdot 2|n| / \pi \phi(2|n|)\right]^{1 / 2} .
$$

We included in Table 1 values of $b_{n}$ so computed for $n=-10,-7,-6,-3,5,6,10$, and 13 , but the last of these is rather crude since the constants $L_{13}\left(2^{k}\right)$ were not available. This difficulty, which was mentioned before, would also exist for $n=22$, 37 , and 58. (This problem is accentuated here since in these three cases $g_{n}$ tends to differ from 1 by more than is usual. This is related to the fact that there are especially many primes of the form $m^{2}+a$ for $a=22,37,58$ [9, p. 326]. Specifically, $g_{13} \approx 1.085$ and $g_{a}$ for the other values of $a$ mentioned would be even larger. Where- 
as, on the contrary, if $m^{2}+a$ has relatively few primes, such as for $a=11,14$, we have values particularly close to 1 . Thus, $g_{11} \approx 1.019$ and $g_{14} \approx 1.009$.)

For $n=-14$, and generally for any $n$ of types $a_{7}$ and $a_{6}$ in Table 3, one could compute $B_{n}(x)$ from $O_{n}(x)$ as in (29). This is so since 2 is in $P_{0}^{\prime}$ and $E_{n}(x)=$ $B_{n}(x / 2)$ as before. But for $n=6$, say, one has the more intricate recurrences:

$$
E_{6}(x)=B_{2,3}(x / 2), \quad E_{2,3}(x)=B_{6}(x / 2) .
$$

To compute $B_{6}(x)$, therefore, $O_{6}(x)$ does not suffice. One must also investigate the second class, and compute $O_{2,3}(x)$ too, whether one wishes to, or not. (The crosscomputation of $B_{6}(x)$ and $B_{2,3}(x)$ from columns of data $O_{6}(x)$ and $O_{2,3}(x)$ is an operation a little like lacing shoes.)

We include in Table 5 values of $B_{6}(x), B_{2,3}(x), B_{10}(x)$, and $B_{2,5}(x)$. One notes at once that the two classes are closely equinumerous for both $n=6$ and $n=10$. Algorithmicly speaking, the recurrence computation just described tends to bring this about, for if $B_{6}(x)>B_{2,3}(x)$, say, then $E_{6}(2 x)<E_{2,3}(2 x)$, and that tends to ('ompensate for the previous excess.

The interesting investigation suggests itself to attempt to prove that all complex zeros of $L_{-2}(s)$ and $L_{3}(s)$ are of the form $a_{i}+i t_{i}$, with $a_{i}<\theta<1$ for some $\theta$ and all $t_{i}<T$, by utilizing the difference $B_{6}(x)-B_{2,3}(x)$ for all $x<X$. If this were successful, there would be a distinct gain, for the computation of this latter difference is very simple, while the direct investigation of the $L$ functions requires elaborate transcendental calculations.

Before leaving this section we note that $B_{n}(x)(\log x)^{1 / 2} / b_{n} x$ converges to 1 somewhat more slowly for $n=6$ and 10 than for $n=1$. The data implies that $c_{6}\left(c_{10}\right)$ is about $34 \%(37 \%)$ larger than $c_{1}$.

Of perhaps greater interest is the following observation. If $p$ is prime, it is well known that the period $\rho$ of the regular continued fraction for $p^{1 / 2}$ is odd or even according as $p \equiv 1$ or $p \equiv-1(\bmod 4)$. Further, the $\rho$ th convergent, $P_{\rho} / Q_{\rho}$, in the first case satisfies

$$
P_{\rho}^{2}-p Q_{\rho}^{2}=-1
$$

But Table 4 calls attention to the less known result that if $p \equiv 3(\bmod 8)$ as in type $a_{3}$, then $\rho=4 k+2$ and

$$
P_{2 k+1}^{2}-p Q_{2 k+1}^{2}=-2
$$

while if $p \equiv 7(\bmod 8)$ as in type $a_{6}$, then $\rho=4 k$ and

$$
P_{2 k}^{2}-p Q_{2 k}^{2}=+2 .
$$

8. Class Number 2 Continued, Other Square-Free $n$. Analogous to types (b) and (c) in Section 3 are 9 other cases of class number 2. These are summarized in Table 6. We spare the reader the details here. Suffice it to say that (6) is again valid, the two classes are again disjoint, and $b_{n}$ is again computed by $(27)$ with the additional factor of $\frac{1}{2}$. We have computed no examples here. 
TABLE 6

Other Variations, Class No. 2

\begin{tabular}{|c|c|c|c|c|c|}
\hline Type & $n$ & $F_{0}$ & $F_{1}$ & $P_{0}^{\prime}$ & $P_{1}^{\prime}$ \\
\hline $\begin{array}{l}b_{1} \\
b_{1} \\
b_{1} \\
b_{1} \\
b_{2} \\
c_{1} \\
c_{1} \\
c_{2} \\
c_{3}\end{array}$ & $\begin{array}{l}-93 \\
-77 \\
-69 \\
-21 \\
-85 \\
-57 \\
-33 \\
-65 \\
+15\end{array}$ & $\begin{array}{l}u^{2}-93 v^{2} \\
u^{2}-77 v^{2} \\
u^{2}-69 v^{2} \\
u^{2}-21 v^{2} \\
u^{2}-85 v^{2} \\
u^{2}-57 v^{2} \\
u^{2}-33 v^{2} \\
u^{2}-65 v^{2} \\
u^{2}+15 v^{2}\end{array}$ & $\begin{array}{l}93 u^{2}-v^{2} \\
77 u^{2}-v^{2} \\
69 u^{2}-v^{2} \\
21 u^{2}-v^{2} \\
5 u^{2}-17 v^{2} \\
57 u^{2}-v^{2} \\
33 u^{2}-v^{2} \\
5 u^{2}-13 v^{2} \\
3 u^{2}+5 v^{2}\end{array}$ & $\begin{array}{r}31 \\
11 \\
3 \\
7 \\
19 \\
3 \\
-\end{array}$ & $\begin{array}{l}3 \\
7 \\
23 \\
3 \\
5,17 \\
8,3 \\
8,11 \\
8,5,13 \\
8,3,5\end{array}$ \\
\hline
\end{tabular}

9. Class Number 2 with $n=4 n_{0}$; Splitting, Nonsplitting, and Overlapping Classes. Eight of the fourteen cases here derive from class number 1 cases $u^{2}+n_{0} v^{2}$, namely: $n=-8,8,-52,-20,12,-68,28$, and 16, where the corresponding $n_{0}$ ('ases have been discussed in Section 3. Five others derive from class number 2 cases in Table 3, namely: $n=-76,-44,-12,-92$, and -28 . The interesting remaining case, $n=-32$, we will return to in the sequel.

We have already (inadvertently) come across the case $n=12$. The form $u^{2}+3 v^{2}$ represents 3 and primes of the form $6 k+1$. If $v$ is even the prime is of the form $12 k+1$ while if $v$ is odd the prime is either of the form $12 k+7$ or the single prime 3 . If we confine ourselves to odd numbers, the two forms, $u^{2}+12 v^{2}$ and $3 u^{2}+4 v^{2}$ behave precisely as in our previous class number 2 cases, including the facts that $O_{3,4}(x) \sim O_{12}(x)$ (as already noted on page 559), that the two classes are disjoint, that a formula such as (6) is again valid, as is also the relation

$$
O_{3,4}(x)-O_{12}(x)=o\left(\frac{x}{(\log x)^{m}}\right) \text { for any } m .
$$

The even numbers represented by $u^{2}+12 v^{2}$ and $3 u^{2}+4 v^{2}$, on the contrary, are identical, since in either case $u$ must be even. Therefore, such a number equals $4\left(r^{2}+3 s^{2}\right)$ for arbitrary $r$ and $s$, and we have the equation

$$
E_{12}(x)=E_{3,4}(x)=B_{3}(x / 4)=E_{3}(x) .
$$

Since $O_{3}(x) \sim 3 E_{3}(x)$, it easily follows that

$$
B_{12}(x) \sim B_{3,4}(x)=\frac{b_{12} x}{(\log x)^{1 / 2}}\left[1+\frac{c_{12}}{\log x}+O\left(\frac{1}{\log ^{2} x}\right)\right]
$$

where $b_{12}=\frac{5}{8} b_{3}$ as in Table 1 .

Three other important cases here are $n= \pm 8$ and 16 . These behave rather similarly to $n=12$ except for the somewhat different behavior of the even numbers represented. This difference reflects the related difference concerning factors of 2 in the cases $n_{0}=3, \pm 2$, and 4 .

Specifically, for $n_{0}= \pm 2,4$, we find that the primes $u^{2}+n_{0} v^{2}$ split in two classes depending upon whether $v$ is even or odd. Thus, we find that these primes, and also all odd numbers, fall into the following arithmetic progressions: 


\begin{tabular}{c|c|c|c}
\hline$v$ & $n_{0}=4$ & $n_{0}=2$ & $n_{0}=-2$ \\
\hline $\begin{array}{l}8 k+1 \\
\text { even }\end{array}$ & $\begin{array}{c}8 k+1 \\
8 k+3\end{array}$ & $\begin{array}{l}8 k+1 \\
8 k+7\end{array}$ \\
\hline
\end{tabular}

Hence, the odd numbers given by the two forms

$$
u^{2}+4 n_{0} v^{2}, \quad(2 u+v)^{2}+n_{0} v^{2}
$$

again are disjoint, equinumerous, and have the same asymptotic behavior. We have written the second form here in such a way as to make it clear that if $n_{0}$ is even and $(2 u+v)^{2}+n_{0} v^{2}$ is odd, then $2 u+v$ and $v$ are also odd. In particular, for $n_{0}=4$, we have

$$
O_{16}(x) \sim O_{4,4,5}(x) \sim \frac{1}{2} O_{4}(x) .
$$

Again, as before, the two forms represent identical even numbers, and we have

$$
E_{4 n_{0}}(x)=B_{n_{0}}(x / 4)
$$

in all these cases. But, for $n_{0}= \pm 2, u^{2}+4 n_{0} v^{2}$ now represents only one-half of the evens represented by $u^{2}+n_{0} v^{2}$, while, for $n_{0}=4$, one may verify that $u^{2}+16 v^{2}$ represents three-quarters of the even numbers of the form $u^{2}+4 v^{2}$. Specifically, the evens of the form $u^{2}+16 v^{2}$ comprise products of any odd number $u^{2}+v^{2}$ times 4,16 , $32,64,128$, or any higher power of 2 . It is clear, in fact, that in the even numbers $u^{2}+4 v^{2}$ we lose the even factor $2^{1}$, in $u^{2}+16 v^{2}$ we further lose $2^{3}$, in $u^{2}+64 v^{2}$ we further lose $2^{5}$, etc.

In the cases $n= \pm 8,16$ we therefore again find (6) valid with the relations

$$
b_{ \pm 8}=\frac{1}{2} b_{ \pm 2}, \quad b_{16}=\frac{7}{16} b_{1}
$$

as in Table 1. (The interested reader can also compute $c_{16}$.)

In Table 7 we have included data for $B_{12}(x)$ and $B_{16}(x)$ together with their related forms. We again record the redundancies:

$$
B_{12}(x)+B_{3,4}(x)=B_{3}(x)+B_{3}(x / 4)
$$

and the more complicated

$$
B_{16}(x)+B_{4,4,5}(x)=B_{1}(x)-B_{1}\left(\frac{x}{2}\right)+2 B_{1}\left(\frac{x}{4}\right)-2 B_{1}\left(\frac{x}{8}\right)+2 B_{1}\left(\frac{x}{16}\right) .
$$

We also note that in Table 7, as in Table 5, the principal form

$$
u^{2}+n v^{2}
$$

usually, but not always, leads its related form in population. This phenomenon is clearly somewhat similar to the Chebyshev phenomena, cf. [16], but we have not attempted to analyze it, even heuristically.

In contrast to the examples just discussed, consider $n=-12$. Here $n_{0}=-3$ and we already have class number 2 . We now find that the odd numbers of the form $u^{2}-12 v^{2}$ include all of those of the form $u^{2}-3 v^{2}$; there is no splitting. For $v$ 
Table 7

Populations, Other Class No. 2

\begin{tabular}{|c|c|c|c|c|}
\hline$x$ & $B_{12}(x)$ & $B_{3,4}(x)$ & $B_{16}(x)$ & $B_{4,4,5}(x)$ \\
\hline $2^{0}$ & 1 & 0 & 1 & 0 \\
\hline $2^{1}$ & 1 & 0 & 1 & 0 \\
\hline $2^{2}$ & 2 & 2 & 2 & 1 \\
\hline $2^{3}$ & 2 & 3 & 2 & 2 \\
\hline $2^{4}$ & 6 & 5 & 4 & 4 \\
\hline $2^{5}$ & 9 & 9 & 8 & 7 \\
\hline $2^{6}$ & 17 & 16 & 13 & 14 \\
\hline $2^{7}$ & 30 & 29 & 25 & 24 \\
\hline $2^{8}$ & 54 & 53 & 44 & 43 \\
\hline $2^{9}$ & 98 & 98 & 83 & 82 \\
\hline $2^{10}$ & 183 & 181 & 152 & 149 \\
\hline $2^{11}$ & 341 & 341 & 286 & 284 \\
\hline $2^{12}$ & 645 & 640 & 538 & 534 \\
\hline $2^{13}$ & 1220 & 1218 & 1020 & 1015 \\
\hline $2^{14}$ & 2327 & 2321 & 1942 & 1937 \\
\hline $2^{15}$ & 4451 & 4449 & 3725 & 3713 \\
\hline $2^{16}$ & 8555 & 8546 & 7145 & 7136 \\
\hline $2^{17}$ & 16489 & 16482 & 13781 & 13759 \\
\hline $2^{18}$ & 31859 & 31845 & 26627 & 26597 \\
\hline $2^{19}$ & 61717 & 61707 & 51572 & 51537 \\
\hline $2^{20}$ & 119779 & 119760 & 100099 & 100045 \\
\hline $2^{21}$ & 232919 & 232865 & 194633 & 194586 \\
\hline $2^{22}$ & 453584 & 453511 & 379037 & 378987 \\
\hline $2^{23}$ & $\$ 84544$ & 884493 & 739250 & 739161 \\
\hline $2^{24}$ & 1727213 & 1727125 & 1443573 & 1443465 \\
\hline $2^{25}$ & 3376505 & 3376376 & 2822186 & 2821923 \\
\hline $2^{26}$ & 6607371 & 6607207 & 5522889 & 5522689 \\
\hline
\end{tabular}

even in $m=u^{2}-3 v^{2}$, it is clear that $m$ is represented. But for $v$ odd, and therefore $u$ even, we may utilize

$$
m=u^{2}-3 v^{2}=(2 u+3 v)^{2}-3(u+2 v)^{2} .
$$

On the right $u+2 v$ is now even, so that $m$ is still represented. For example:

$$
13=4^{2}-3 \cdot 1^{2}=11^{2}-3 \cdot 6^{2}=11^{2}-12 \cdot 3^{2} \text {. }
$$

Therefore

$$
O_{-12}(x)=O_{-3}(x)
$$

and $n=-12$ still belongs to class number 2 . As before we have

$$
E_{-12}(x)=B_{-3}(x / 4) \text {. }
$$

Whereas Gauss and his contemporaries studied quadratic forms for all $n$, there is some modern tendency to confine oneself to square-free $n$. We must note here, however, that we often find those $n$ which are divisible by squares to be of special interest. Thus, we have seen, for the first time in this section, the phenomena of splitting, nonsplitting and overlapping classes. Further, as we shall see, while some class number 3,4 , or 8 cases, which are of much interest, are difficult to compute 
when they first occur at $n=11,14,21,41$, and 56 , one can study essentially similar behavior for $n=27,20,24,256$, and 96, respectively, and now the computation goes through much more easily since the corresponding Dirichlet series $L_{n}(s)$ are known.

10. Class Number 2 Concluded. For brevity, and to allow us to proceed more quickly to essentially new phenomena, we forego a complete treatment of the previous cases. An interested reader can easily fill the gaps. For example, the fact that class number 2 cases $n_{0}=-23,-19,-11$, and -7 remain class number 2 for $n=4 n_{10}$, just as it did for $n_{0}=-3$, follows from the fact that if

$$
u^{2}-n_{0} v^{2}=\text { odd }, \quad x^{2}-n_{0} y^{2}=1,
$$

with $v$ odd and $u$ even, then

$$
u^{2}-n_{0} v^{2}=\left(x u+n_{0} y v\right)^{2}-n_{0}(y u+x v)^{2},
$$

and now $y u+x v$ is even since the fundamental solutions $x+y \sqrt{ } n_{0}$ are given by $24+5 \sqrt{ } 23,170+39 \sqrt{ } 19,10+3 \sqrt{ } 11$, and $8+3 \sqrt{ } 7$, respectively. Similarly, the remaining nine cases of class number 2 in our range, namely those where $n$ is divisible by an odd square: $n=9,18,-18,-27,-45,-54,25,-50$, and 98 , we also bypass. The reader will have no difficulty in verifying, for example, that $b_{9}=5 b_{1} / 9$ as in Table 1 .

Since our paper is a long one, and its publication has been unduly delayed (the work was mostly done several years ago), we now declare an intermission. In the sequel we shall continue with the more intricate class number 4,8 , and 3 phenomena, and with discussion of some of the topic's mentioned at the end of Section 1.

Applied Mathematics Laboratory

David Taylor Model Basin

Washington, D. C. 20007

1. Daniel Shanks, "The second-order term in the asymptotic expansion of B(x)," Math. Comp., v. 18, 1964, pp. 75-86. MR 28 \#2391.

2. H. H. OstmanN, Additive Zahlentheorie, Zweiter Teil, Springer, Berlin, 1956, p. 84. MR 20 \#5176.

3. R. D. JAMES, "The distribution of integers represented by quadratic forms," Amer. J. Math., v. 60, 1938, pp. 737-744.

4. GoRdON PALL, "The distribution of integers represented by binary quadratic forms," Bull. Amer. Math. Soc., v. 49, 1943, pp. 447-449. MR 4, 240.

5. Paul Bernays, Über die Darstellung von positiven, ganzen Zahlen durch die primitiven, binären quadratischen Formen einer nicht-quadratischen Diskriminante, Diss. Göttingen, 1912.

6. L. E. Dickson, History of the Theory of Numbers, Vol. III, Stechert, New York, 1934, p. 49 .

7. Harald Bohr, Collected Mathematical Works, Vol. III, Dansk Matematisk Forening, Copenhagen, 1952, H. Encyclopaedia Article, p. 841. MR 15, 276.

8. DANiel Shanks \& J. W. Wrench, JR., "The calculation of certain Dirichlet series," Math. Comp., v. 17, 1963, pp. 135-154. Corrigenda, ibid., p. 488. MR $28 \# 3012$.

9. DaNiel Shanks, "On the conjecture of Hardy \& Littlewood concerning the number of primes of the form $n^{2}+a, "$ Math. Comp., v. 14, 1960, pp. 320-332. MR $22 \# 10960$.

10. EDMUND LandaU, "Über die Einteilung der positiven ganzen Zahlen in vier Klassen nach der Mindeszahl der zu ihren additiven Zusammensetzung erforderlichen Quadrate," Arch. der Math. und Physik, (3), v. 13, 1908, pp. 305-312.

11. DANIEL SHANKs, "Polylogarithms, Dirichlet series, and certain constants," Math. Comp., v. 18, 1964, pp. 322-324. MR $30 \# 5460$.

12. TRYGVE NAGELL, Introduction to Number Theory, Wiley, New York, Almqvist \& Wiksell, Stockholm, 1951, pp. 205-206. MR 13, 207.

13. SRinivasa Ramanujan, Collected Papers, Cambridge, 1927, pp. 171-172. 
14. E. LANDAU, "Über die Verteilung der Primideale in den Idealklassen eines algebraischen Zahlkörpers," Math. Ann., v. 63, 1907, pp. 145-204. This paper was utilized by Bernays in his [5]. See also [7, p. 840].

15. C. F. Gauss, Recherches Arithmétiques, reprinted by Blanchard, Paris, 1953. See Paragraph 304 in Section V.

16. Daniel Shanks, "Quadratic residues and the distribution of primes," $M T A C, \mathrm{v} .13$, 1959, pp. 272-284. MR 21 \#7186. 